\title{
Nucleosynthesis in Early Supernova Winds: The Role of Neutrinos
}

\section{R. D. Hoffman}

Lawence Livermore National Laboratory

PO Box 808, L-414 Livermore, CA 94550 USA

E-mail: hoffman21日IInI.gov

J. Pruet

Lawence Livermore National Laboratory

PO Box 808, L-059 Livermore, CA 94550 USA

E-mail: pruetI@IInI.gov

\section{S. E. Woosley}

Department of Astronomy \& Astrophysics

UC Santa Cruz, Santa Cruz, CA 95064 USA

E-mail: woosleyeucolick.org

\section{H.-T. Janka and R. Buras}

Max Plank Institute for Astrophysics

Karl-Schwarzschild-Str. 1, 85741 Garching, Germany

E-mail: thjempa-garching.mpg.de, rburasempa-garching.mpg.de

\begin{abstract}
Nucleosynthesis in early proton-rich neutrino winds is investigated. The inclusion of charged-current neutrino reactions on free nucleons indicates that in conditions extracted from a current supernova model nucleosynthesis will proceed some 40 mass units higher than if they are neglected. An analytical estimate of how high in $\mathrm{Z}$ the nucleosynthesis might proceed as a function of the neutrino flux and the usual parameters defining the expansion (entropy, $Y_{e}$, expansion timescale) is presented as well as the sensitivity of the nucleosynthesis to variations in late time neutron addition and increases to the entropy predicted in the model due to various physical processes that were not included.
\end{abstract}

International Symposium on Nuclear Astrophysics - Nuclei in the Cosmos - IX

CERN, Geneva, Switzerland

25-30 June, 2006

\footnotetext{
${ }^{*}$ Speaker
} 


\section{Introduction}

One of the outstanding unsolved riddles of nuclear astrophysics is the origin of the socalled " $p$-process" nuclei from $A=92$ to 126 . Both the lighter and heavier $p$-process nuclei are adequately produced in the neon and oxygen shells of ordinary Type II supernovae [1], but the origin of these intermediate isotopes, especially ${ }^{92,94} \mathrm{Mo}$ and ${ }^{96,98} \mathrm{Ru}$, has long been mysterious. Here we explore the production of these nuclei in the neutrino-driven wind from a young neutron star. We consider such early times that the wind still contains a proton excess because the rates for $v_{\mathrm{e}}$ and positron captures on neutrons are faster than those for the inverse captures on protons. Following a suggestion by Frohlich et al. [2], we also include the possibility that, in addition to the protons, $\alpha$-particles, and heavy seed, a small flux of neutrons is maintained by anti-neutrino captures on free protons. This flux of neutrons is critical in bridging the long waiting points along the path of the $r p$-process by $(\mathrm{n}, \mathrm{p})$ reactions. Using the unmodified ejecta histories from a recent two-dimensional supernova model by Janka et al. [3], we find synthesis of proton-rich nuclei up to ${ }^{102} \mathrm{Pd}$. However, if the entropy of these ejecta is increased by a factor of two, the synthesis extends to ${ }^{120} \mathrm{Te}$. Still larger increases in entropy, that might reflect the role of magnetic fields [4,5], acoustic energy input [5,6], or Alfven wave dampening [7], neglected in the hydrodynamical model, result in the production of numerous $r-, s-$, and $p$-process nuclei up to $\mathrm{A} \sim 170$, even in winds that are proton-rich.

\subsection{Nucleosynthesis in six Neutrino Wind Trajectories}

Our nucleosynthesis calculations are motivated by conditions drawn from the 2-D 15 solar mass core collapse model of Janka et al. [3]. We derived six wind trajectories from the ejecta [8], the table below summarizes a few pertinent conditions at the end of each nucleosyntheis calculation. The columns are, from left to right, the trajectory number, the electron fraction $\mathrm{Y}_{\mathrm{e}}$, the entropy $s / k_{b}$, the mass fractions for free protons, alpha-particles, the sum of all species heavier than ${ }^{4} \mathrm{He}$, and that of ${ }^{56} \mathrm{Ni}$, the percentage of $\mathrm{X}_{\mathrm{H}}$ that was ${ }^{56} \mathrm{Ni}$, and $\Delta_{\mathrm{n}}$, the number of neutrons produced by anti-electron neutrino capture on free protons per heavy seed created (see section 3). Results from the hot bubble trajectories were not very different when neutrino-induced reactions were included and are not presented in this contribution.

\begin{tabular}{ccccccccc}
\hline \hline traj. & $Y_{e}$ & $s / k_{b}$ & $X(p)$ & $X(\alpha)$ & $X_{H}$ & $X(56 \mathrm{Ni})$ & $\%{ }^{\mathrm{b}}$ & $\Delta_{n}{ }^{\mathrm{c}}$ \\
\hline 1 & 0.539 & 54.8 & 0.078 & 0.614 & 0.307 & 0.244 & 80 & 0.2 \\
2 & 0.548 & 58.0 & 0.095 & 0.714 & 0.190 & 0.135 & 71 & 0.4 \\
3 & 0.551 & 76.7 & 0.101 & 0.822 & 0.075 & 0.043 & 57 & 1.7 \\
4 & 0.551 & 71.0 & 0.102 & 0.796 & 0.101 & 0.063 & 62 & 1.1 \\
5 & 0.556 & 74.9 & 0.113 & 0.831 & 0.054 & 0.025 & 46 & 2.9 \\
6 & 0.558 & 76.9 & 0.115 & 0.840 & 0.043 & 0.014 & 33 & 3.2 \\
\hline
\end{tabular}

Figure 1 shows integrated production factors, P(i), from all six trajectories calculated both with and without neutrino captures on free nucleons. Nucleosynthesis stops some 40 mass units lower if neutrinos are excluded. The $p$-nuclei of ${ }^{84} \mathrm{Sr},{ }^{94} \mathrm{Mo},{ }^{96,98} \mathrm{Ru}$, and ${ }^{102} \mathrm{Pd}$ are co-produced in amounts that might explain their abundance in the sun, although ${ }^{92} \mathrm{Mo}$ is problematic due the uncertain (small) proton separation energy of ${ }^{91} \mathrm{Rh}$.. 

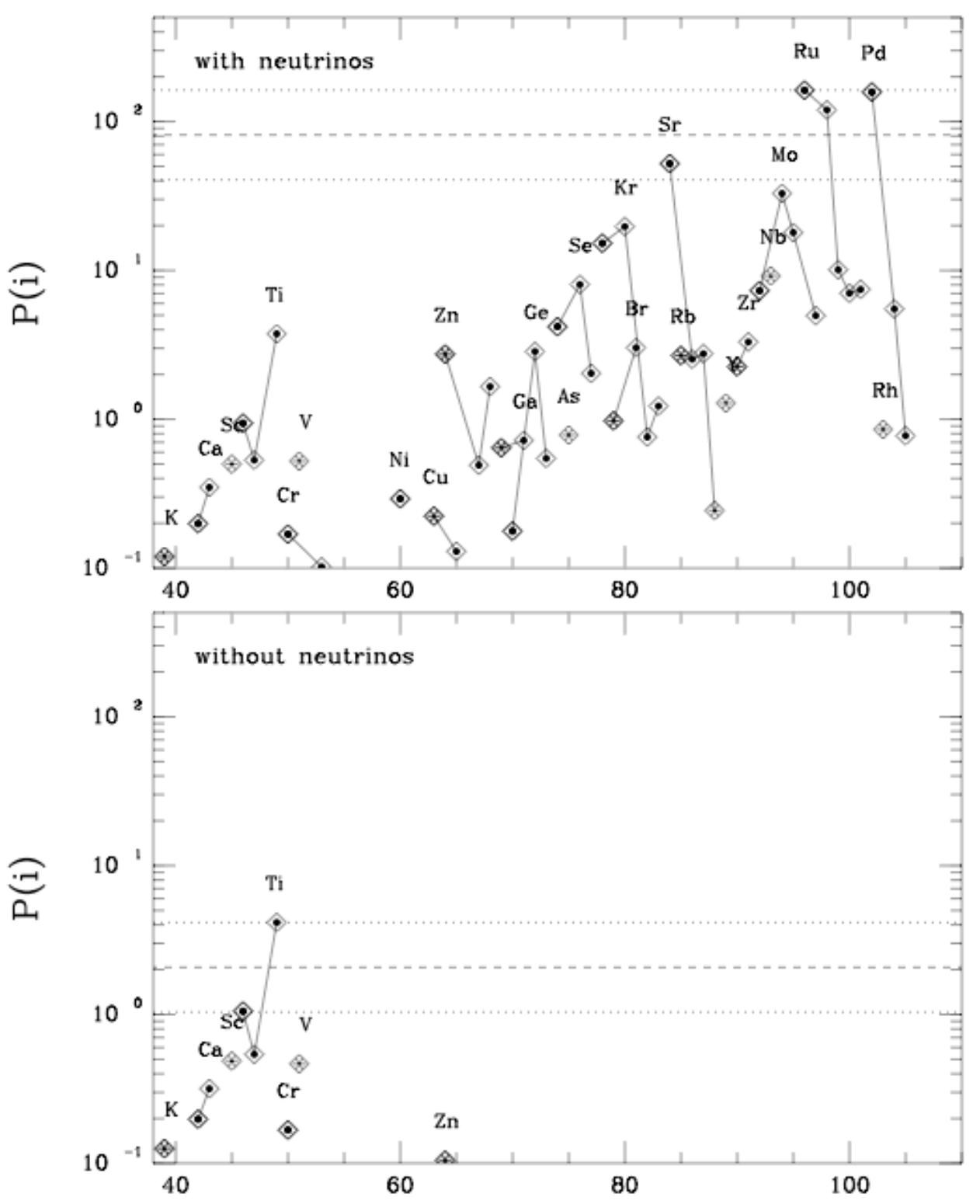

A

Figure 1. Sown are the production factors $\mathrm{P}(\mathrm{i})$, from the combined nucleosynthesis in the six wind trajectories described in this section. The production factors are defined as the sum of the isotopic mass fractions in the wind divided by their mass fraction in the sun weighted by the mass of the material in the wind trajectory divided by the total mass ejected $\left(13.5 \mathrm{M}_{\text {sun }}\right)$ in the supernova model. 


\section{Character of the Nucleosynthesis}

Qualitatively, the nucleosynthesis occurs in three, possibly four, stages. First, neutrons combine with protons in the outgoing wind making $\alpha$-particles and heavy seed nuclei [8]. In the second stage $\left(1.5<\mathrm{T}_{9}<3\right),(\mathrm{p}, \gamma)$ and $(\mathrm{n}, \mathrm{p})$ reactions carry the flow along the $\mathrm{Z}=\mathrm{N}$ line past the classical $r p$-process waiting points, leading to the production of the intermediate $p$-nuclei between $\mathrm{Kr}$ and $\mathrm{Pd}$. In the third stage $\left(\mathrm{T}_{9}<1.5\right)$ the charged-particle reactions freeze-out, and neutrons, continually produced by anti-neutrino capture onto protons, are assimilated by the heavy seed distribution, moving material rapidly towards stability. The fourth stage, if it occurs, happens under conditions when many neutrons are created per heavy seed nucleus at low temperatures over a prolonged neutrino irradiation, leading to potential production of $r$-process nuclei. We explore in the following three figures the sensitivity of the nucleosynthesis to variations in the entropy per baryon predicted by the supernova model in trajectory 6 . Such entropies could be realized if physical processes such as magnetic fields or acoustic energy input were included in the supernova model.

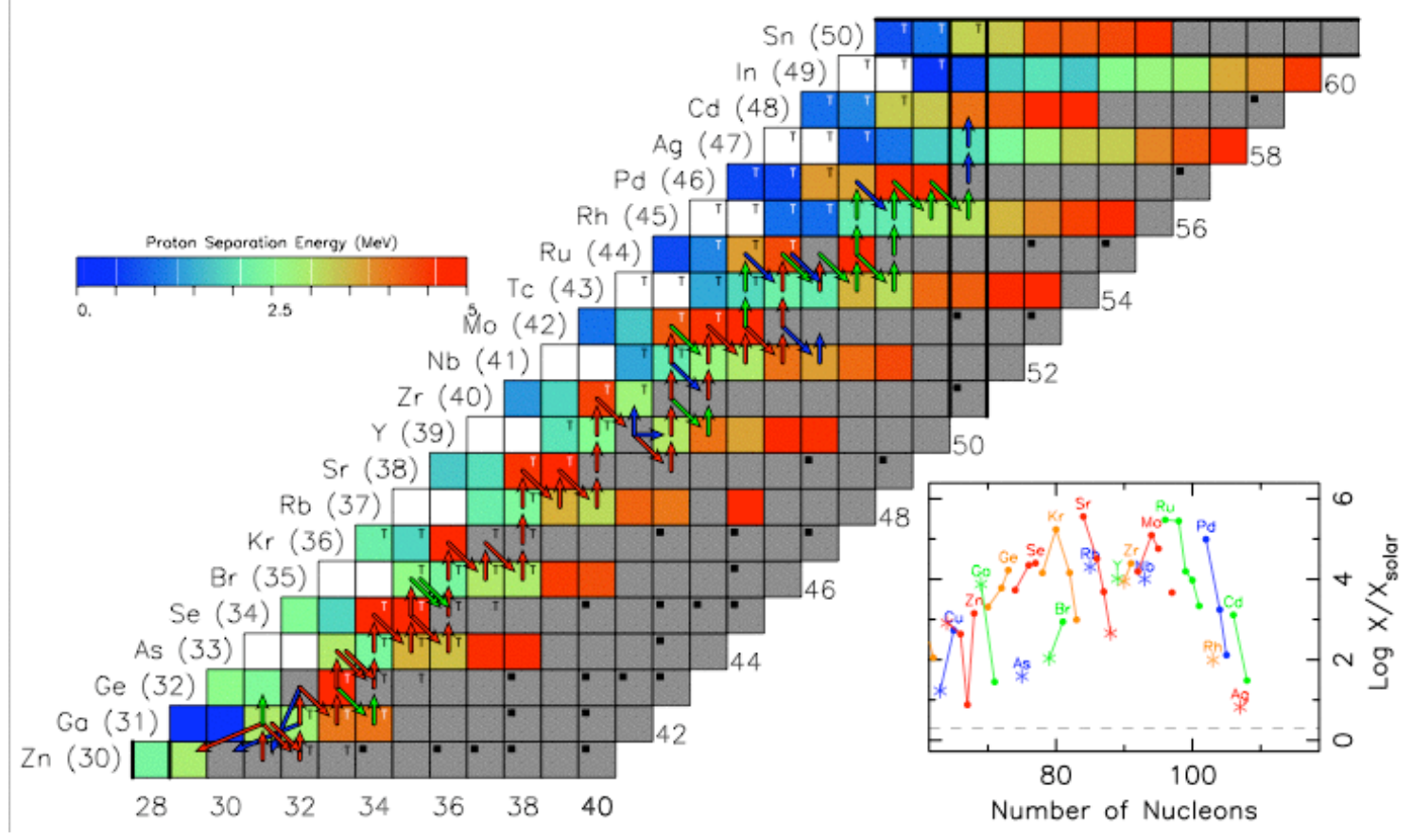

Figure 2. Net nuclear flows in the (Z,N)-plane from $\mathrm{Zn}$ to $\mathrm{Sn}$ in the unmodified wind outflow of trajectory 6 . Here $\mathrm{t}=0.35 \mathrm{sec}$ since $\mathrm{T}_{9 \mathrm{i}}=9, \mathrm{~T}_{9}=2.05, \rho=2.7 \times 10^{4} \mathrm{~g} \mathrm{~cm}^{-3}\left(\mathrm{~s} / \mathrm{k}_{\mathrm{b}} \sim 77\right)$, and $\mathrm{Y}_{\mathrm{e}}=$ 0.562. Net flows are plotted in three strengths: red (strong), green (intermediate) and blue (weak). Stable species are represented by a filled black square in the upper left corner. Each nucleus is color coded according to the legend by the value of its proton separation energy. A "T" is plotted in the upper right-hand corner for nuclei with a binding energy that was extrapolated from measured masses. The classical $r p$-process waiting points ${ }^{64} \mathrm{Ge},{ }^{68} \mathrm{Se},{ }^{72} \mathrm{Kr}$, and ${ }^{76} \mathrm{Sr}$ are bypassed by $(\mathrm{n}, \mathrm{p})$ reactions with the neutrons provided by anti-neutrino captures on free protons. Due to the small (and uncertain) proton spearation energy of ${ }^{91,92} \mathrm{Rh}$, the proton capture flow up the $\mathrm{N}=48$ isotone is diverted to $\mathrm{N}=50$, breaking the sequence of abundant even$\mathrm{Z}$ even-N nuclei that serve as decay parents for their $p$-process daughters up to this point. Thus, ${ }^{92} \mathrm{Mo}$ (the most abundant $p$-isotope), seems to be the only one with an odd- $\mathrm{Z}$ odd-N parent. 

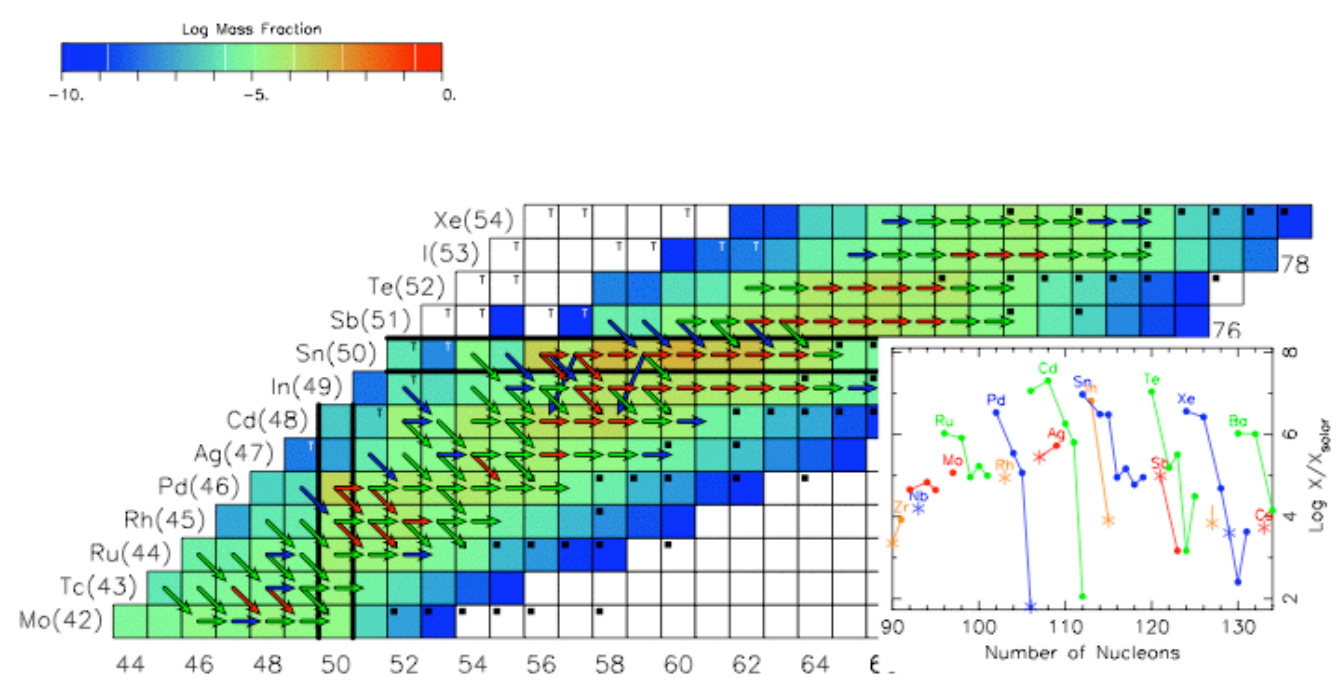

Figure 3. Net nuclear flows from Mo to Xe in the modified $\left(\mathrm{s} / \mathrm{k}_{\mathrm{b}} \sim 150\right)$ wind trajectory 6 . Here $\mathrm{t}$ $=2.15 \mathrm{sec}, \mathrm{T}_{9}=0.93, \rho=9.7 \times 10^{2} \mathrm{~g} \mathrm{~cm}^{-3}$, and $\mathrm{Y}_{\mathrm{e}}=0.56$. The flows are depicted exactly as in the figure above, but now each isotope is color coded according to the legend by the logarithm of its mass fraction. The charged particle reactions have frozen out, leaving $(n, p)$ and $(n, \gamma)$ reactions to carry the flow rapidly towards stability (before the onset of weak decay). This allows $p$-nuclei like ${ }^{106} \mathrm{Cd},{ }^{112} \mathrm{Sn},{ }^{120} \mathrm{Te}$, and ${ }^{124,126} \mathrm{Xe}$ to be made as themselves via neutron capture reactions. Note, just as the $r$-process can contribute to nuclei made by the $s$-process that are unshielded against $\beta^{-}$decay, so too can the $v$-rp process considered here contribute to $s$-nuclei that are unshielded from $\beta^{+}$decay. This suggests a new production scenario designation, " $p, s$ ", for some nuclei previously identified as " $r, s$ " or even "s-only".

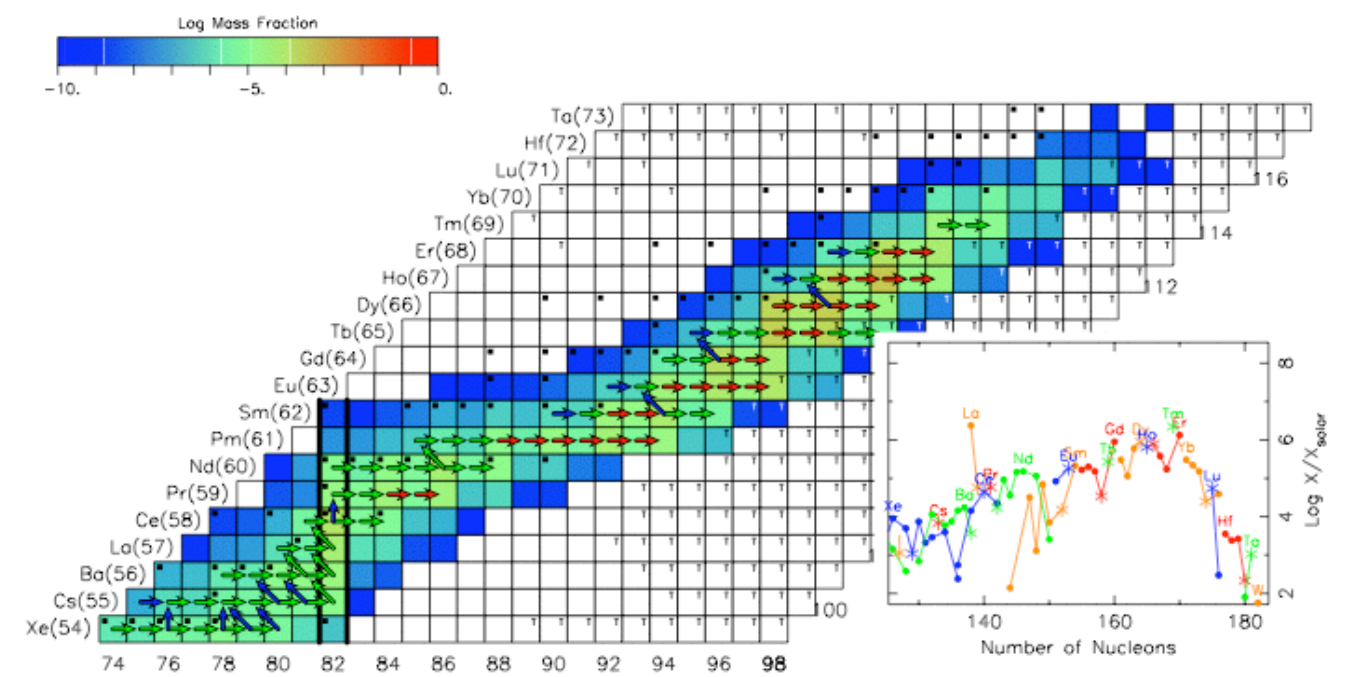

Figure 4. Net nuclear flows from Xe to Ta in the modified $\left(\mathrm{s} / \mathrm{k}_{\mathrm{b}} \sim 220\right)$ wind trajectory 6 . Here $\mathrm{t}$ $=0.58 \mathrm{sec}, \mathrm{T}_{9}=1.81, \rho=5.4 \times 10^{3} \mathrm{~g} \mathrm{~cm}^{-3}$, and $\mathrm{Y}_{\mathrm{e}}=0.561$. In the region of the rare earth elements 
the major flows have already moved material through the valley of stability, producing neutronrich species which after decay will populate the $r, s$ - and $r$-process isotopes of elements from Sm to Hf. The production of ${ }^{138} \mathrm{La}$ remains high due to its proximity to the closed neutron shell at $\mathrm{N}$ $=82$ and the fact that it is shielded from both $\beta^{-}$and $\beta^{+}$decay.

\section{3. $\Delta_{\mathrm{n}}$ - a Basic Parameter}

In the limit where anti-neutrino captures on protons becomes important, nuclei are pushed to higher mass and isospin via $(\mathrm{n}, \mathrm{p})$ and $(\mathrm{n}, \gamma)$ reactions. To estimate how far the nucleosynthesis can proceed we have identified a key dimensionless parameter, $\Delta_{\mathrm{n}}$, which represents a measure of the number of neutrons produced by neutrino captures on free protons $\left(X_{p}\right)$ compared to the number of heavy seed nuclei made $\left(\mathrm{X}_{\mathrm{H}}\right)$ for various combinations of neutrino luminosity, expansion time scale, entropy, and electron fraction $[5,9]$ :

where

$$
\Delta_{n}=\frac{X_{p} n_{\bar{v}}}{X_{H} / \bar{A}} \approx 60 \frac{\left(2 Y_{e}-1\right) n_{\bar{v}}}{1-X_{\alpha}-X_{p}}
$$

$$
n_{\bar{v}}=\int \lambda_{\bar{v}} d t
$$

and

$$
\lambda_{\bar{v}} \approx 0.06 \frac{L_{\bar{v}_{e}}}{10^{52} \mathrm{erg} / \mathrm{sec}} \frac{T_{\bar{v}_{e}}}{4 \mathrm{MeV}}\left(\frac{10^{8} \mathrm{~cm}}{r}\right)^{2}
$$

In figure 5 we present the influence of the weak production of neutrons at low temperatures. The top panel shows nucleosynthesis in trajectory 6 with its normal outflow velocity $\left(4 \times 10^{8} \mathrm{~cm} \mathrm{~s}^{-1}\right)$, characterized by the weak production $\left(\Delta_{\mathrm{n}} \sim \mathrm{few}\right)$ of neutrons per heavy seed nucleus. All species are made as radioactive proton-rich progenitors. In the middle panel the radial history at late times is kept closer to the neutron star and $\Delta_{\mathrm{n}} \sim 5$. Production of some $p$ nuclei $\left({ }^{98} \mathrm{Ru}\right.$ and $\left.{ }^{114} \mathrm{Xe}\right)$ is greatly reduced as the flows have now carried material into the valley of stability. The situation is much like that depicted in Figure 3. The bottom panel shows a further modification to the radius vs. time history with $\Delta_{\mathrm{n}} \sim 20$. Many neutrons are captured at low temperatures and all major species now have neutron-rich progenitors, allowing for the production the $r$-only nuclei ${ }^{110} \mathrm{Pd},{ }^{116} \mathrm{Cd}$, and ${ }^{122,124} \mathrm{Sn}$. This is similar to the situation depicted in Figure 4.

For the production of intermediate mass $p$-nuclei, these results suggest two constraints on $\Delta_{\mathrm{n}}$. In proton-rich wind outflows synthesizing $p$-nuclei with mass near $130, \Delta_{\mathrm{n}}$ must be of order 10. At low temperatures, when charged particle reactions have frozen out but neutrons are still readily absorbed, minimal destruction of $p$-nuclei requires $\Delta_{\mathrm{n}}<$ a few. It may be difficult, however, to have ejecta that is both cold enough and close enough to the neutron star to experience the necessary neutrino irradiation. For further details, see Pruet et al. [10]. 

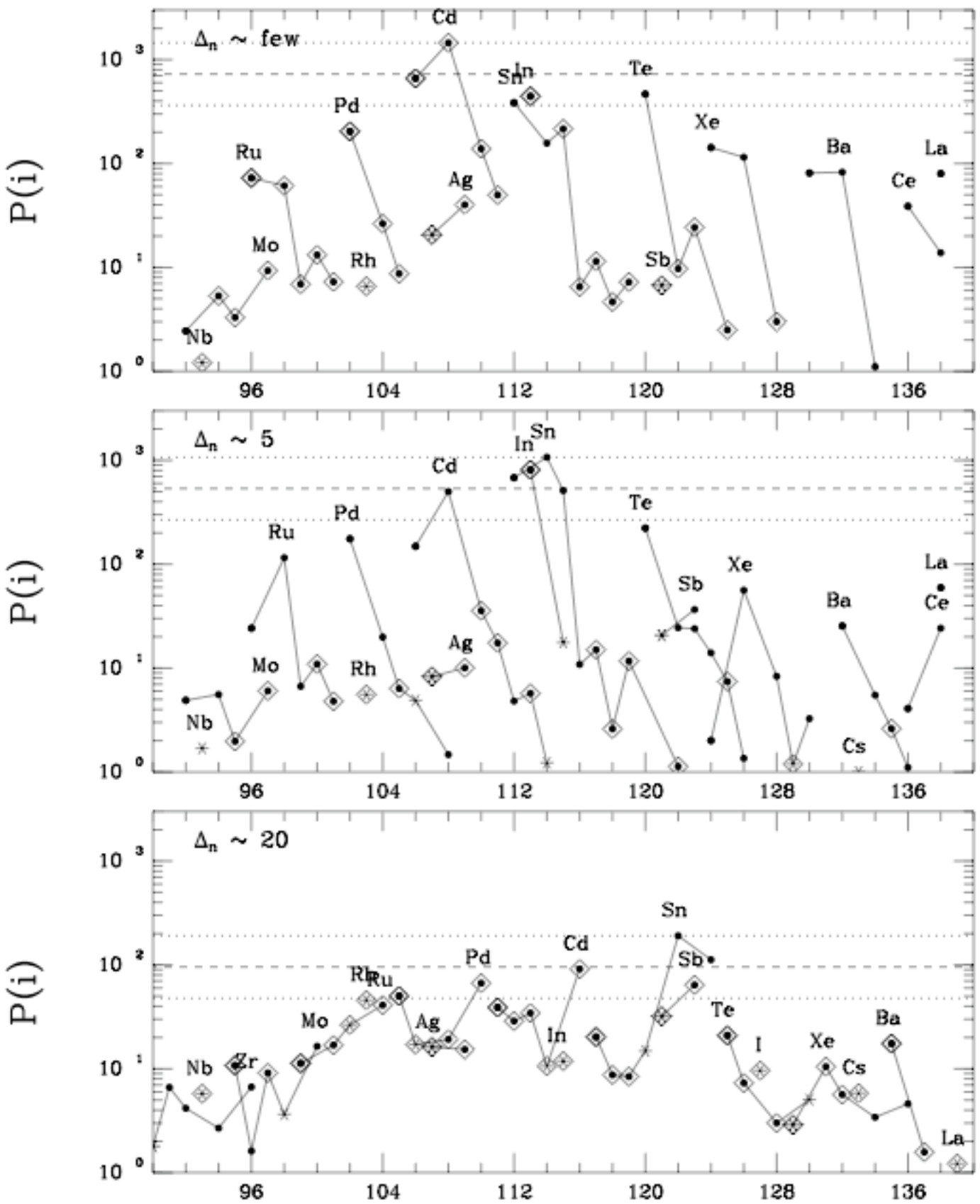

A

Figure 5. Sensitivity of the nucleosynthesis in trajectory 6 to the addition of neutrons at late times as represented by the dimensionless parameter $\Delta_{\mathrm{n}}$. The character of the nucleosynthesis produced changes drmatically from intermediate proton-rich $p$-process nuclei, to heavier nuclei made as themselves in the valley of stability, to finally neutron-rich nuclei, some of which are traditionally attributed to the $r$-process. 


\section{Acknowledgments}

This work was performed under the auspices of the U.S. Department of Energy by the University of California Lawrence Livermore National Laboratory under contract No. W-7405Eng-48. It was also funded by the DOE-OS SciDAC program (DC-FC02-01ER41176), the National Science Foundation (AST-02-06111), and NASA (NAG5-12036). In Germany, funding for H.-T.J. and R.B. was provided by the Research Center for Astroparticle Physics (SFB 375) and the Transregional Collaborative Research Center for Gravitational Wave Astronomy (SFB-Transregio 7). Reviewed and released at LLNL as UCRL-PROC-222936.

\section{References}

[1] S. E. Woosley \& M. Howard, The p-process in supernovae. ApJS, 36, 285 (1978)

[2] C. Fröhlich, G. Martínez-Pinedo, M. Liebendörfer, F.-K. Thielemann, E. Bravo, W. R. Hix, K.-H. Langanke, \& N. T. Zinner, Neutrino-induced Nucleosynthesis of A>64 Nuclei: the vp-process. PRL 96, 142502 (2006)

[3] H.-Th. Janka, R. Buras, \& M. Rampp, The Mechanism of Core-collapse Supernovae and the Ejection of Heavy Elements. Nucl. Phys. A718, 269 (2003)

[4] T. Thompson, Magnetic Protoneutron Star Winds and r-Process Nucleosynthesis. ApJ 585, L33 (2003)

[5] Y.-Z. Qian \& S. E. Woosley, Nucleosynthesis in Neutrino-driven Winds. I. The Physical Conditions. ApJ 471, 331 (1996).

[6] A. Burrows, E. Livne, L. Dessart, C. D. Ott, J. Murphy, A New Mechanism for Core-Collapse Supernova Explosions. ApJ 640, 878 (2006)

[7] T. Suzuki \& S. Nagataki, Alfvén Wave-driven Proto-Neutron Star Winds and r-Process Nucleosynthesis. ApJ 628, 914 (2005)

[8] J. Pruet, S. E. Woosley, R. Buras, H.-Th..Janka, \& R. D. Hoffman, Nucleosynthesis in the Hot Convective Bubble in Core-Collapse Supernovae. ApJ 623, 325 (2005)

[9] R. D. Hoffman, S. E. Woosley, \& Y.-Z. Qian, Nucleosynthesis in Neutrino-driven Winds. II. Implications for Heavy Element Synthesis ApJ 482, 951 (1997)

[10] J. Pruet, R. D. Hoffman, S. E. Woosley, H.-Th..Janka, \& R. Buras, Nucleosynthesis in Early Supernova Winds. II. The Role of Neutrinos. ApJ 644, 1028 (2006) 